\title{
Preliminary Measurements of an Integrated Prototype of the CubeSat Ambipolar Thruster
}

\author{
Timothy A. Collard* and J. P. Sheehan ${ }^{\dagger}$ \\ University of Michigan, Ann Arbor, MI, 48109, USA
}

\begin{abstract}
An integrated engineering unit of the CubeSat Ambipolar Thruster (CAT) was tested at power levels up to $43 \mathrm{~W}$. With the incorporation of a closed loop cooling system CAT was able to operate for hours, and steady state measurements indicated that the electrical efficiency was $\sim 50 \%$. Plume oscillations measured with a Langmuir probe showed a maximum peak-to-peak floating potential oscillation of $150 \mathrm{mV}$ and implying that RF compensation of probes is not required. Angular ion current density profiles were measured a various distances downstream of the thruster exit plane, demonstrating the ion current closely follows the magnetic nozzle geometry. A parameter study of the peak ion density and plume divergence half angle was conducted. The peak current density increased and the plume divergence half angle decreased with decreasing propellant mass flow rate, while the peak current density increased and the plume divergence half angle was insensitive to increasing input power. Indirect thrust measurements indicated a thrust improvement over the corresponding cold gas thrust. However, these results are degraded by the prevalence of scattering collisions due to high background pressure during operation. A discussion on considerations for indirect thrust measurements is included. Improvements to the CAT architecture and future experiments are discussed.
\end{abstract}

\section{Nomenclature}

$\dot{m}_{X e} \quad$ Propellant mass flow rate, $\mathrm{mg} / \mathrm{s}$

$\eta_{E} \quad$ Electrical efficiency

$I_{D C} \quad$ Input DC current, $\mathrm{A}$

$P_{D C} \quad$ Input DC power, $\mathrm{W}$

$P_{R e j} \quad$ Heat rejected through cooling system, W

$t_{\text {fire }} \quad$ Thruster firing time, min

$T_{\text {Pelt }} \quad$ Temperature of top surface of Peltier stack, $\mathrm{K}$

$T_{W B} \quad$ Temperature of the interface between Peltier stack and water block, $\mathrm{K}$

$V_{D C} \quad$ Input DC voltage, $\mathrm{V}$

\section{Introduction}

$\mathrm{D}$

UE to standardization, modularization, and shared launch costs the CubeSat architecture, based on multiples or fractions of a cube with $10 \mathrm{~cm}$ sides (one $10 \mathrm{~cm}$ cube is $1 \mathrm{U}$ ), has reduced costs and accelerated mission development cycles. These advantages have made CubeSats attractive options to both commercial and scientific groups that are interested in a wide range of missions types, from imaging to satellite health monitoring to space weather. Many of these missions require a level of satellite maneuverability and, therefore, a propulsion system. The exact $\Delta V$ requirements are mission dependent, but can range from less than $10 \mathrm{~m} / \mathrm{s}$ to over $1000 \mathrm{~m} / \mathrm{s}$.

CubeSat propulsion is not as advanced as most other small satellite technologies due to difficulties in miniaturizing existing propulsion technologies. With increasing nanosatellite capabilities there is interest in $\Delta V$ intensive maneuvers, including station-keeping, deorbiting, constellation phasing, and dynamic orbit

\footnotetext{
*Ph.D. Candidate, Department of Aerospace Engineering, University of Michigan, and AIAA Student Member

${ }^{\dagger}$ Assistant Research Scientist, Department of Aerospace Engineering, University of Michigan, and AIAA Member.
} 
changes. ${ }^{1}$ There are a number of monopropellant and bipropellant systems under development, but these are generally limited to thrust $\leq 1 \mathrm{~N}$ and $\Delta V$ of $10 \mathrm{~s}$ of $\mathrm{m} / \mathrm{s}^{2-11}$ For the moderate $\Delta V$ required for these types of maneuvers or for extended missions electric propulsion is required. A number of electric thrusters are being developed with published $\Delta V$ capabilities approaching $500 \mathrm{~m} / \mathrm{s}^{12-23}$

The CubeSat Ambipolar Thruster (CAT) is designed to enable high $\Delta V(\geq 1000 \mathrm{~m} / \mathrm{s})$ missions. These missions include geostationary orbit insertion from geotransfer orbit, interplanetary exploration, long-lived low altitude orbits. ${ }^{24}$ This article describes the the initial performance and plume measurements of an integrated engineering unit of the CAT, which was designed to identify places for design optimization and to prepare for full integration into a flight unit.

\section{The CubeSat Ambipolar Thruster}

\section{A. Thruster}

CAT is an electrodeless helicon thruster that generates thrust by expanding plasma through a magnetic nozzle. As depicted in Figure 1a the propellant is injected into the plasma liner where radio frequency (RF) power is coupled via a helical half-twist antenna, resulting in ionization. The plasma then exits the ionization region through a converging-diverging magnetic nozzle generated by permanent magnets. The magnetic field is parallel to the plasma liner walls, limiting wear and plasma recombination at the wall. Downstream the plasma detaches from the magnetic nozzle and thrust is generated via pressure on the magnets and the momentum of the ions. A photograph of the integrated thruster, produced by Phase Four, Inc., can be found in Figure 1b. The mesh located behind the thruster was installed to protect the power processing unit (PPU) from spurious secondary plasma formation caused by the antenna coupling to the high neutral density in the chamber due to the high flow rates during stable operation. While CAT can be integrated into a CubeSat form factor, including a propellant management system and a battery bank, for the experiments discussed herein it was controlled by external DC power supplies and a $50 \mathrm{sccm}$ mass flow controller with uncertainty of $\pm 1 \%$ to allow for precise control over input parameters.

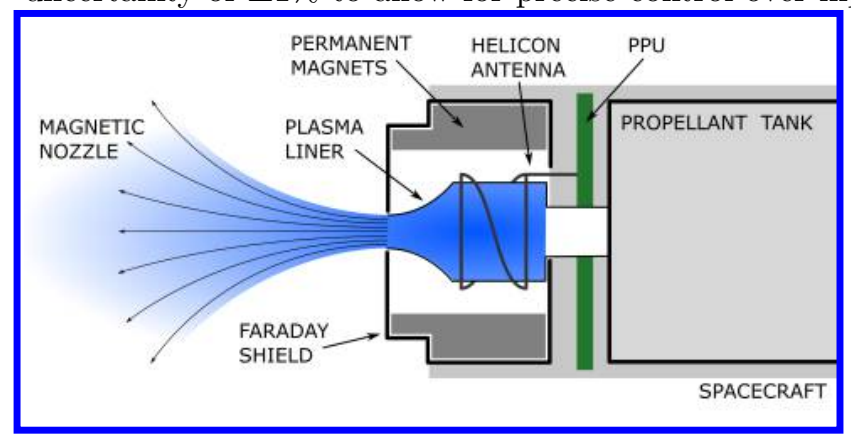

(a)

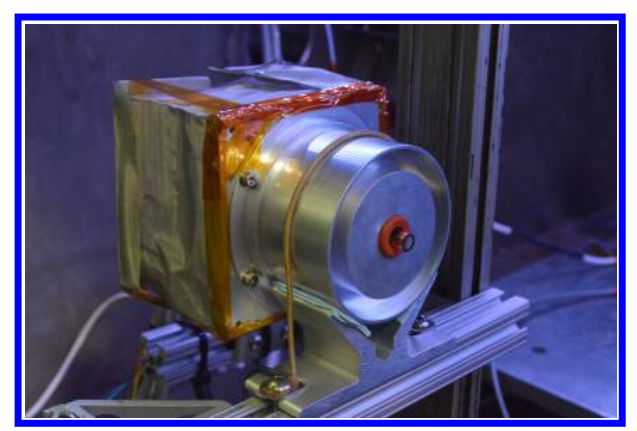

(b)

Figure 1: (a) A notional schematic of CAT. (b) A photograph of the engineering unit of CAT. The mesh behind the thruster is meant to limit damage to the PPU from spurious plasma formation caused by the high pack pressures observed during firing.

\section{B. Power Processing Unit}

The integrated CAT utilizes a dedicated PPU to convert DC to RF power and couple RF power to the helical half-twist antenna and resulting plasma. The PPU was capable of delivering up to $50 \mathrm{~W}$ of power at 9.96 $\mathrm{MHz}$. Currently, impedance matching is achieved through fixed capacitors and a $100 \mathrm{kHz}$ programmatic frequency setpoint range; future iterations will include variable frequency matching hardware to allow for matching after ignition. The $50 \mathrm{~W}$ threshold was chosen based on the capabilities of state-of-the-art small satellite power buses. ${ }^{25}$ The PPU is located in close proximity to the helical half-twist antenna to reduce line losses and facility interactions caused by capacitive coupling to the vacuum chamber. The housekeeping and telemetry stage of the PPU requires $8-12 \mathrm{~V}$ DC power, and continuously transmits telemetry. The housekeeping stage was operated at a nominal $10 \mathrm{~V}$ by an external DC power supply. During startup the $\mathrm{RF}$ generation stage of the PPU switches from a high voltage, low current state to a low voltage, high current mode, requiring a wide input voltage and current range. To accommodate these requirements the 
RF generation stage of the PPU was backed by an external $60 \mathrm{~V}-10$ A DC power supply during the experiments discussed in this paper.

\section{Experimental Apparatus}

\section{A. Vacuum Facility}

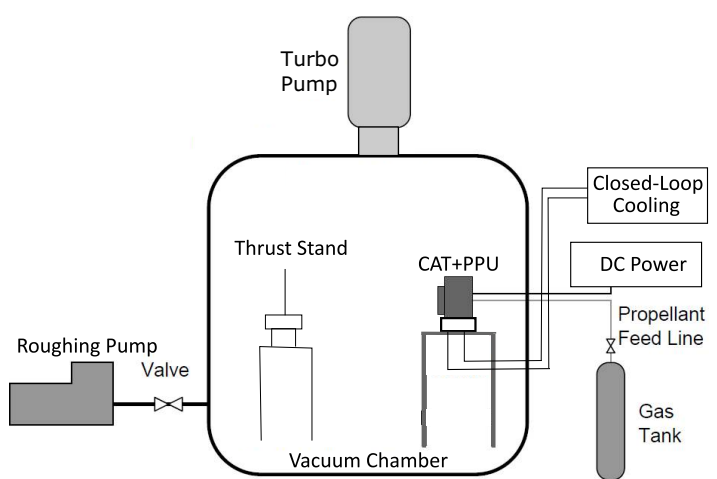

(a) The thrust measurement experimental setup.

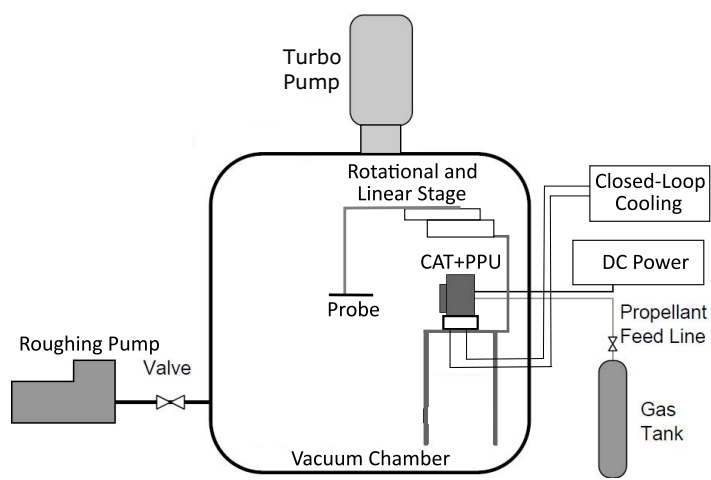

(b) The experimental setup for measuring ion current angular profiles.

Figure 2: The experimental configurations.

These experiments were conducted in the Junior Test Facility connected to the Large Vacuum Test Facility at the University of Michigan. Junior is a 3 meter long, by 1 meter diameter stainless steel clad vacuum chamber capable of achieving base pressures of $3 \times 10^{-6}$ Torr and a background pressure of $5 \times 10^{-4}$ Torr during thruster operation at propellant flow rates up to $3.5 \mathrm{mg} / \mathrm{s}$. The chamber was maintained at high vacuum by a turbopump with a nominal pumping speed of $1650 \mathrm{~L} / \mathrm{s}$ on nitrogen or $800 \mathrm{~L} / \mathrm{s}$ on xenon.

\section{B. Thermal Management System}

During initial checkout of the integrated CAT unit a dangerously rapid rise in thruster temperature limited operation to $\leq 1$ minute, indicating a significant amount of waste heat generation. The waste heat is caused by a number of factors, including shifts in matching impedance after ignition and increasing losses in the RF generation stage components with increasing temperature. To extend thruster operation times and estimate the waste heat generation a thermal management system was implemented. As illustrated in Figure 3, CAT was mounted to a Peltier cooler that was backed by a closed water cooling loop to maintain a constant temperature reservoir and to reject heat external to the vacuum chamber via a forced convection heat exchanger. The temperature difference across the Peltier stack was measured by a pair of temperature sensors with

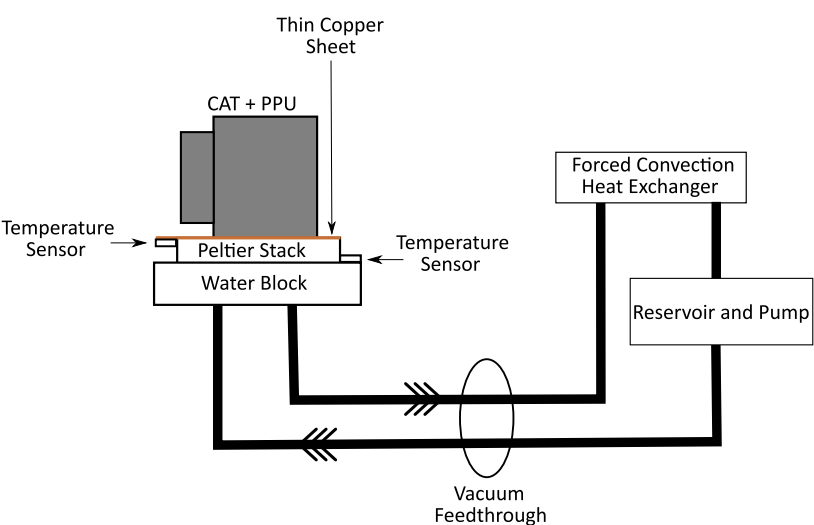

Figure 3: A functional diagram of the thermal management system used during engineering testing of CAT. $\pm 0.1^{\circ} \mathrm{C}$ precision. The linear relationship between the temperature difference and the heat pumped through the Peltier stack, for a given Peltier DC current input, was used to estimate the waste heat generation of CAT at steady state, assuming that conduction through the closed-loop cooling dominates radiative heat transfer. Based on simple blackbody radiation calculations the radiative heat transfer is $<2 \%$ of the estimated waste heat generated by CAT, validating this assumption. To reduce uncertainties in the waste heat estimates the integrated closed-loop cooling system temperature difference was calibrated using a high-power resistor in a vacuum as a dummy load. To allow for margin and future higher power testing the cooling system was sized to safely reject $120 \mathrm{~W}$. 


\section{C. $\mu \mathbf{N}$ Thrust Stand}

With expected thrust in the low $\mathrm{mN}$ range a novel $\mu \mathrm{N}$ nulltype torsional balance thrust stand with a sensitivity of 10 $\mathrm{nN}$ capable of supporting a $10 \mathrm{~kg}$ mass (corresponding to a fully integrated $6 \mathrm{U}$ CubeSat) was developed, Figure 4. In order to keep the stand level a counterweight was placed at the opposite end of the stand. As a force was applied to the stand an optical displacement sensor measured the displacement. This displacement signal was fed into a PID controller that applied current to the nulling electromagnetic coil. The electromagnetic coil current was correlated to thrust through calibration of the stand using a series of known masses at a known radial position on the stand. Stand sensitivity could be adjusted by changing the location of the calibration, then equating the calibration and nulling torques. Drift in the zero-point of the thrust stand was accounted for by in-situ recalibration between thruster firings and during postprocessing.

For initial prototype testing the thruster in Figure 4 was replaced with a $10 \mathrm{~cm}$ diameter graphoil momentum target that was electrically isolated from the stand by an alumina rod fixed to a mounting bracket. As depicted in the diagram in Figure 2a the thruster was located $15 \mathrm{~cm}$ from the momentum target and fired into the target due to the numerous required connections for the integrated thruster subsystems. CAT, with fully integrated subsystems, will be mounted directly to the thrust stand for future performance testing.

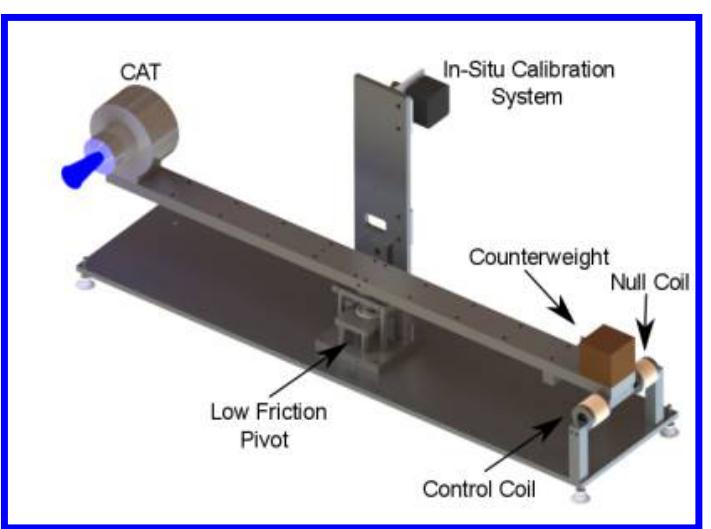

Figure 4: A model of the $\mu \mathrm{N}$ thrust stand used to measure integrated thruster performance.

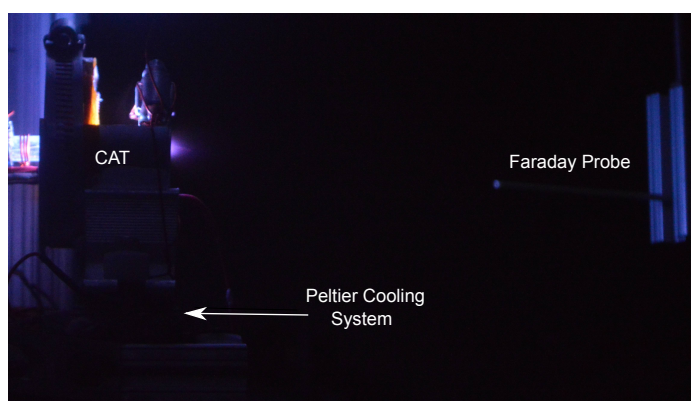

Figure 5: A photograph of CAT firing with the Faraday probe visible.

\section{Faraday Probe}

A nude planar Faraday probe was swept azimuthally from $-90^{\circ}$ to $90^{\circ}$ at constant radii between 5 and $20 \mathrm{~cm}$ from the thruster exit. The collection area was biased sufficiently negative such that the probe was saturated and collected primarily ion current. As shown in Figure $2 \mathrm{~b}$ the desired probe position could be selected by a combination of the linear and rotational stages. Figure 5 shows CAT operating in the middle of an angular Faraday probe sweep.

\section{Results and Discussion}

\section{A. Thermal Measurements}

Due to the operational limitations of CAT caused by thermal runaway it was imperative that an estimate of the waste heat generation be measured and used to inform future design iterations. By implementing the closed loop cooling system the firing time was extended from minutes to hours. At the end of each long duration firing the thruster temperature was measured with a temperature sensor embedded near the half-twist antenna, with the steady state thruster temperature within $55-85^{\circ} \mathrm{C}$, depending on operating condition. The temperature limit of the PPU components was $100^{\circ} \mathrm{C}$, so the thruster operating duration was not limited by thermal effects.

To measure the waste heat generated and the electrical efficiency CAT was operated in a current-limited mode at $1.75 \mathrm{~A}$, detailed in Table 1 . The cause of the variations in the voltage required to drive the current in the antenna is unclear, but could be due to changes in the PPU components from thermal cycling or changes in power coupling to the plasma during startup. Throughout the reported firings the estimated electrical efficiency, or the ratio of power deposited into the plasma to total input power, was determined by Eq. 1,

$$
\eta_{E}=\frac{V_{D C} I_{D C}-P_{R e j}}{V_{D C} I_{D C}}=\frac{P_{D C}-P_{R e j}}{P_{D C}}
$$


Table 1: The operating conditions, firing time, and the cooling system parameters used to infer an estimate of the waste heat generation. The Peltier cooler was powered at $6 \mathrm{~A}$ for all cases $\left(I_{\text {Pelt }}=6 \mathrm{~A}\right)$.

\begin{tabular}{ccccccccc}
$\dot{m}_{X e}(\mathrm{mg} / \mathrm{s})^{*}$ & $V_{D C}(\mathrm{~V})^{*}$ & $I_{D C}(\mathrm{~A})^{*}$ & $P_{D C}(\mathrm{~W})^{\ddagger}$ & $T_{P e l t}(\mathrm{~K})^{\dagger}$ & $T_{W B}(\mathrm{~K})^{\dagger}$ & $P_{\text {Rej }}(\mathrm{W})^{\ddagger}$ & $\eta_{E}(\%)^{\ddagger}$ & $t_{\text {fire }}(\mathrm{min})^{\dagger}$ \\
\hline 3.0 & 16.29 & 1.75 & 28.5 & 272.5 & 296.1 & 15.0 & 47.3 & 487 \\
3.0 & 15.30 & 1.75 & 26.8 & 272.1 & 269.9 & 13.7 & 48.7 & 377 \\
3.0 & 15.47 & 1.75 & 27.1 & 270.5 & 297.5 & 11.4 & 58.1 & 263 \\
3.0 & 14.78 & 1.75 & 25.9 & 267.8 & 295.3 & 10.8 & 58.2 & 199
\end{tabular}

* Measured input thruster parameter.

$\dagger$ Measured cooling system parameter.

¥Value inferred via measured parameters.

where $\eta_{E}$ is the estimated electrical efficiency, $V_{D C}$ is the input DC voltage, $I_{D C}$ is the input DC current, $P_{D C}$ is the DC input power, and $P_{R e j}$ is the heat rejected through the cooling system. For Peltier coolers the power rejected through the stack is a linear function dependent on the temperature difference across the stack and the DC current powering the stack, or $P_{R e j}\left(T_{W B}-T_{P e l t}, I_{P e l t}\right)$. This linear function was determined through calibration of the cooling system. For all cases this resulted in an estimated electrical efficiency of $\sim 50 \%$, indicating a place for future design improvement. Interestingly, there appears to be a trend of decreasing estimated electrical efficiency with increasing firing time, but this is too little data to confirm this trend. The cause of this apparent trend is unclear.

\section{B. Potential Oscillations within the Plume}

Prior to constructing electrostatic probes to measure plume properties oscillations within the plasma must be measured. If the plasma oscillation amplitudes are too large the probes must be RF compensated to reduce the fluctuations such that accurate measurements can be made. In this experiment the oscillations within the plume were measured by a floating planar Langmuir probe connected to an oscilloscope sampling at $1 \mathrm{GHz}$. The floating potential oscillations at $5 \mathrm{~cm}$ downstream of the thruster exit plane is shown in Figure 6a, and the corresponding fast Fourier transform (FFT) in Figure 6b. Similar results were obtained at $5 \mathrm{~cm}$ intervals up to $20 \mathrm{~cm}$ downstream. Figure 7 shows that the floating potential fluctuations attenuated with increasing distance from the thruster exit plane. With a maximum measured fluctuation of $\sim 150 \mathrm{mV}$ useable electrostatic probe traces can be obtained without complex RF compensation.

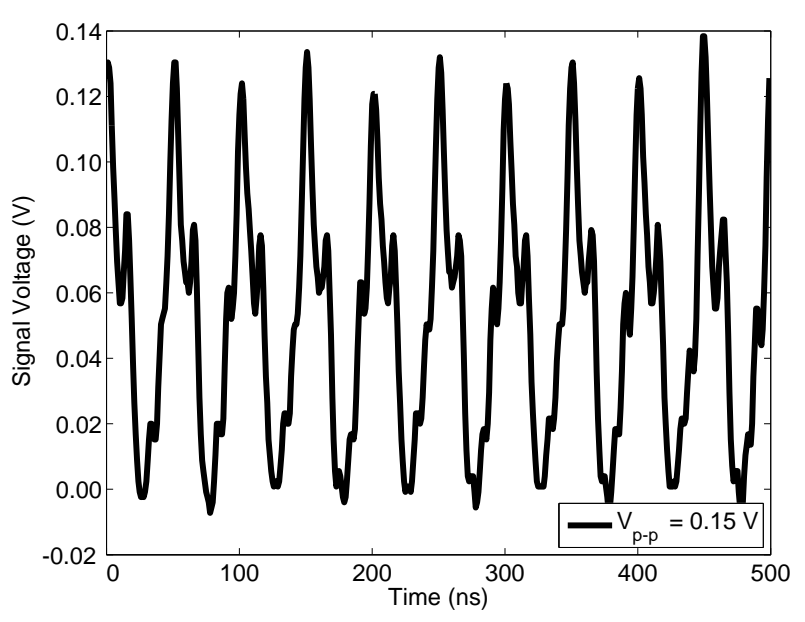

(a)

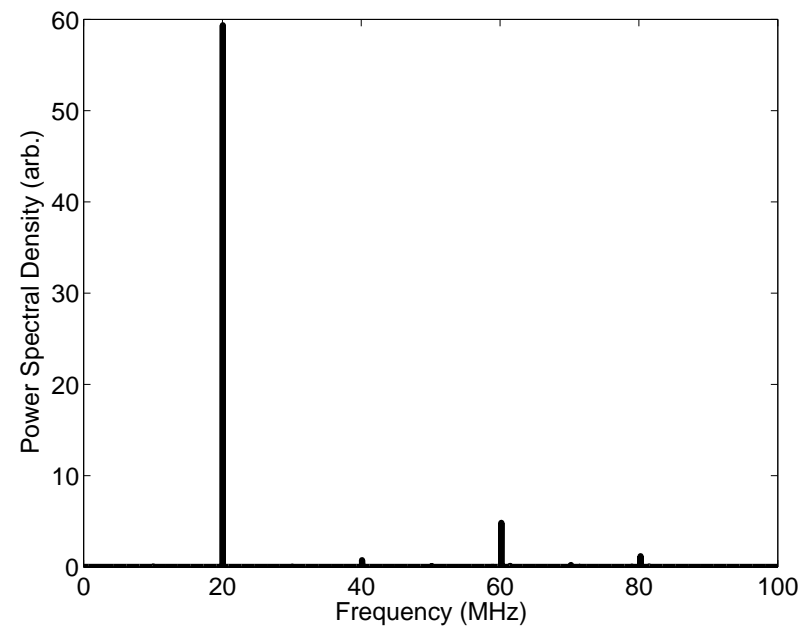

(b)

Figure 6: (a) The measured floating potential signal on a planar Langmuir probe at $5 \mathrm{~cm}$ downstream of the thruster exit plane at a $28.2 \mathrm{~W}$ DC operating condition. (b) The corresponding FFT used to decompose the signal in into its dominant frequency components. Note that there is a minor peak in the FFT at $10 \mathrm{MHz}$, but is $\sim 0.08 \%$ of the magnitude of the $20 \mathrm{MHz}$ peak. 


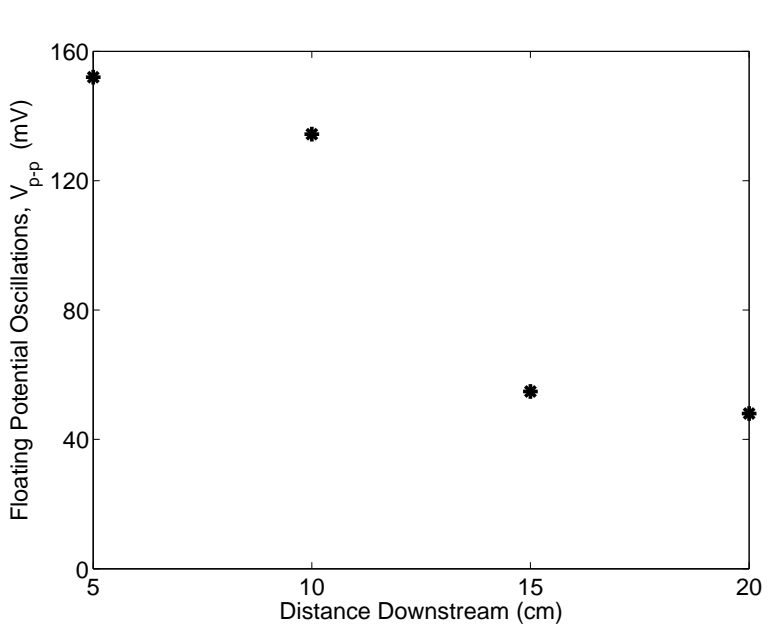

Figure 7: The attenuation in the peak-to-peak voltage in the signal collected by the planar Langmuir probe as the distance from the thruster exit plane increased. This attenuation is consistent with the decrease in floating potential as the plume expands through the magnetic nozzle.

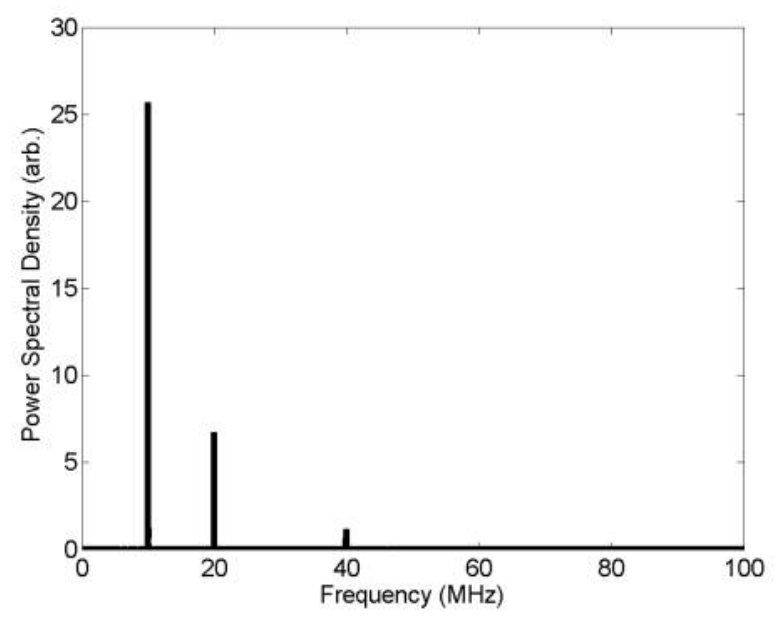

Figure 8: An FFT of the frequencies output by the antenna.

However, the FFT's revealed an interesting phenomenon: even though CAT operates at $\sim 10 \mathrm{MHz}$, the dominant peaks in the power spectral density are the even harmonics of this fundamental frequency. Within the FFT's there is a minor peak at $\sim 10 \mathrm{MHz}$, but it is $\sim 0.08 \%$ of the magnitude of the $\sim 20 \mathrm{MHz}$ dominant peak. To confirm that the CAT supplied a $\sim 10 \mathrm{MHz}$ signal, the antenna frequency was directly measured by the oscilloscope. As expected, the measured antenna frequency showed that the fundamental frequency dominated, but the first several harmonics were also present, as seen in Figure 8 . To further investigate the cause of the dominance of the even harmonics the plasma frequency was checked. To reach a plasma frequency of $20 \mathrm{MHz}$ the plasma density must be $\sim 1.25 \times 10^{11} \mathrm{~m}^{-3}$, which is on the low side for an expanding plasma. If the plasma frequency was being measured the dominant peak should increase as the density decreased downstream of the thruster. The expected decrease in density was not observed, making measurement of the plasma frequency an unlikely cause. The investigation of this phenomenon is ongoing, with effects of the absence of rematching after ignition and plasma instabilities being considered.

\section{Ion Current Density}

The initial plasma diagnostics included a planar Faraday probe swept from $-90^{\circ}-90^{\circ}$ at a constant distance from the thruster exit plane from $5-20 \mathrm{~cm}$ to determine the angular current density profiles. Figure 9 shows an ion current density profile at $5 \mathrm{~cm}$ with the CAT input power of $25.6 \mathrm{~W}$ and propellant flow rate of $3 \mathrm{mg} / \mathrm{s}$. The peaked nature of the curve suggests the presence of an ion beam; if no beam was present the profile would be much flatter across the region inside the magnetic nozzle, in the $5 \mathrm{~cm}$ case within $\sim 20^{\circ}$ of the peak of the profile. The offset from $0^{\circ}$, or the thruster centerline, is likely due to a combination of uncertainty in the positioning of the Faraday probe and imperfections in the permanent magnets generating the magnetic nozzle, resulting in a slight vectoring of the nozzle.

An initial investigation on the effect of propellant mass flow rate and DC input power is shown in Figure 10. For the varying propellant mass flow rate case the CAT was operated in a constant-current limited mode at $2.05 \mathrm{~A}$, but as the propellant flow rate increased the total DC power draw of the thruster decreased. However, Figure 10(a) clearly shows that decreasing the propellant flow rate increases the peak ion current density. This is likely caused by a combination of higher total power available and higher coupled power density. For the $2.5 \mathrm{mg} / \mathrm{s}$ case the peak current density was $305 \%$ of the $3 \mathrm{mg} / \mathrm{s}$ case, with an input power increase of $6 \%$ over the corresponding power at $3 \mathrm{mg} / \mathrm{s}$. The $3 \mathrm{mg} / \mathrm{s}$ case, while not as drastic an improvement over the $3.5 \mathrm{mg} / \mathrm{s}$ case, shows an peak ion current density of $105 \%$ the $3.5 \mathrm{mg} / \mathrm{s}$ case, with a corresponding increase in input power of only $2 \%$. While the overall effect on performance cannot be definitively concluded 
without more probe data, this suggests that at lower propellant mass flow rates the CAT ionizes more of the propellant and/or significantly increases the ion velocity, both of which are general indicators of improved performance.

For the varying power case the propellant flow rate rate was held at a constant $3 \mathrm{mg} / \mathrm{s}$ while the current limit input to the CAT was increased. The trend of increasing peak current density with increasing power holds with the exception of the lowest power case. It is unclear if that result is anomalous or if it is a real effect; further data is required to determine the cause of this local minimum in peak current density. For this particular propellant flow rate the effect of input power on the peak current density is not as dramatic as the effect of propellant flow variation.

The limits of two methods of calculating the divergence half angle are also shown in Figure 9. One commonly used method of determining the plume divergence half angle is by finding where $95 \%$ of the current resides, but this tends to over estimate the divergence angle. Another simple way is by taking the full width half maximum. Results of both of these methods are shown in Figure 11 . In addition to the increase in peak current density with decreasing propellant mass flow rate, the divergence half angle decreases, indicating a much tighter beam. In contrast, the divergence angle, and resulting beam tightness, appears to be constant with varying input power, suggesting power minimally effects the plume divergence. It is important to note that the reported half angles are estimates because the probe could not distinguish between beam and axial current, as required to use the more accurate momentum-weighted method of determining plume divergence developed by Brown, et. al. ${ }^{26,27}$

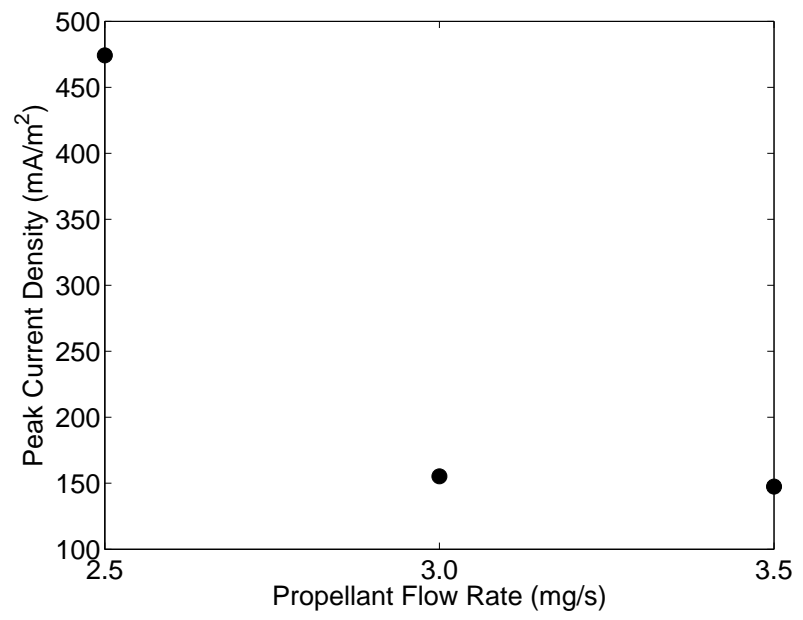

(a)

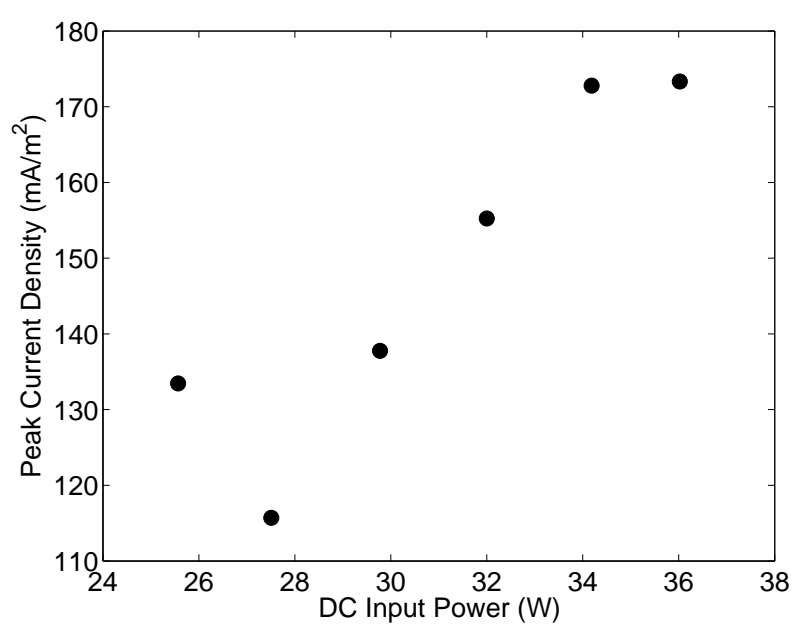

(b)

Figure 10: (a) The change in the peak ion current density as a function of propellant flow rate. The thruster was operated in a constant, current-limited mode at $2.05 \mathrm{~A}$. (b) The variation in the peak ion current density as a function of input DC power. The thruster was operated at a constant propellant flow rate of $3 \mathrm{mg} / \mathrm{s}$. Note that for both figures the error bars are smaller than the markers. 
Full Width Half Maximum

$\Delta$ Integration

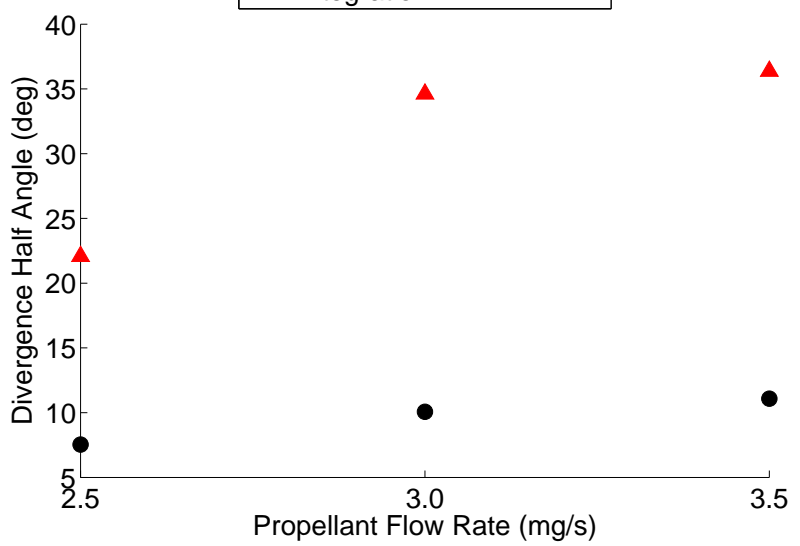

(a)

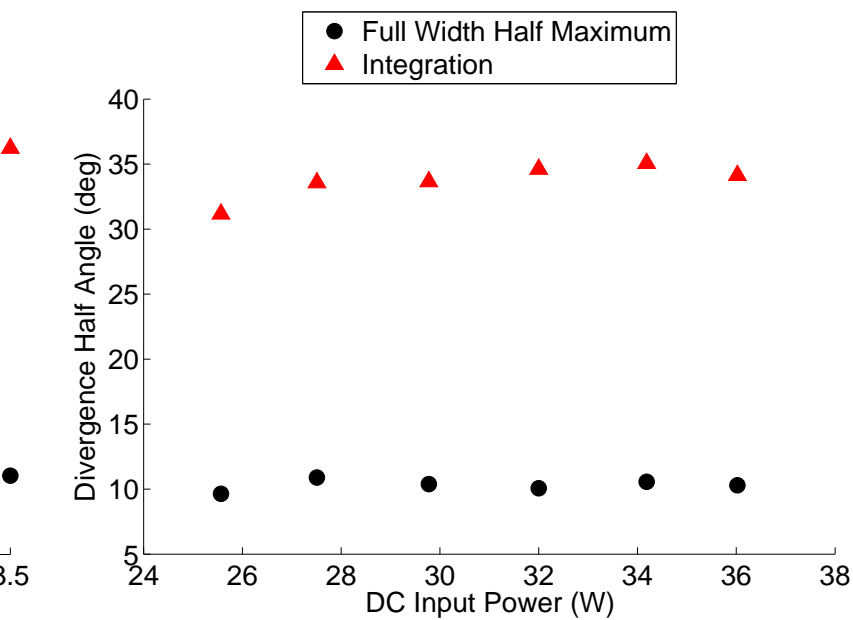

(b)

Figure 11: (a) The variation in the divergence half angle as a function of propellant flow rate. The thruster was operated in a constant current-limited mode at $2.05 \mathrm{~A}$. (b) The variation in divergence half angle as a function of input DC power. The thruster was operated at a constant propellant flow rate of $3 \mathrm{mg} / \mathrm{s}$. Note that for both figures the error bars are smaller than the markers.

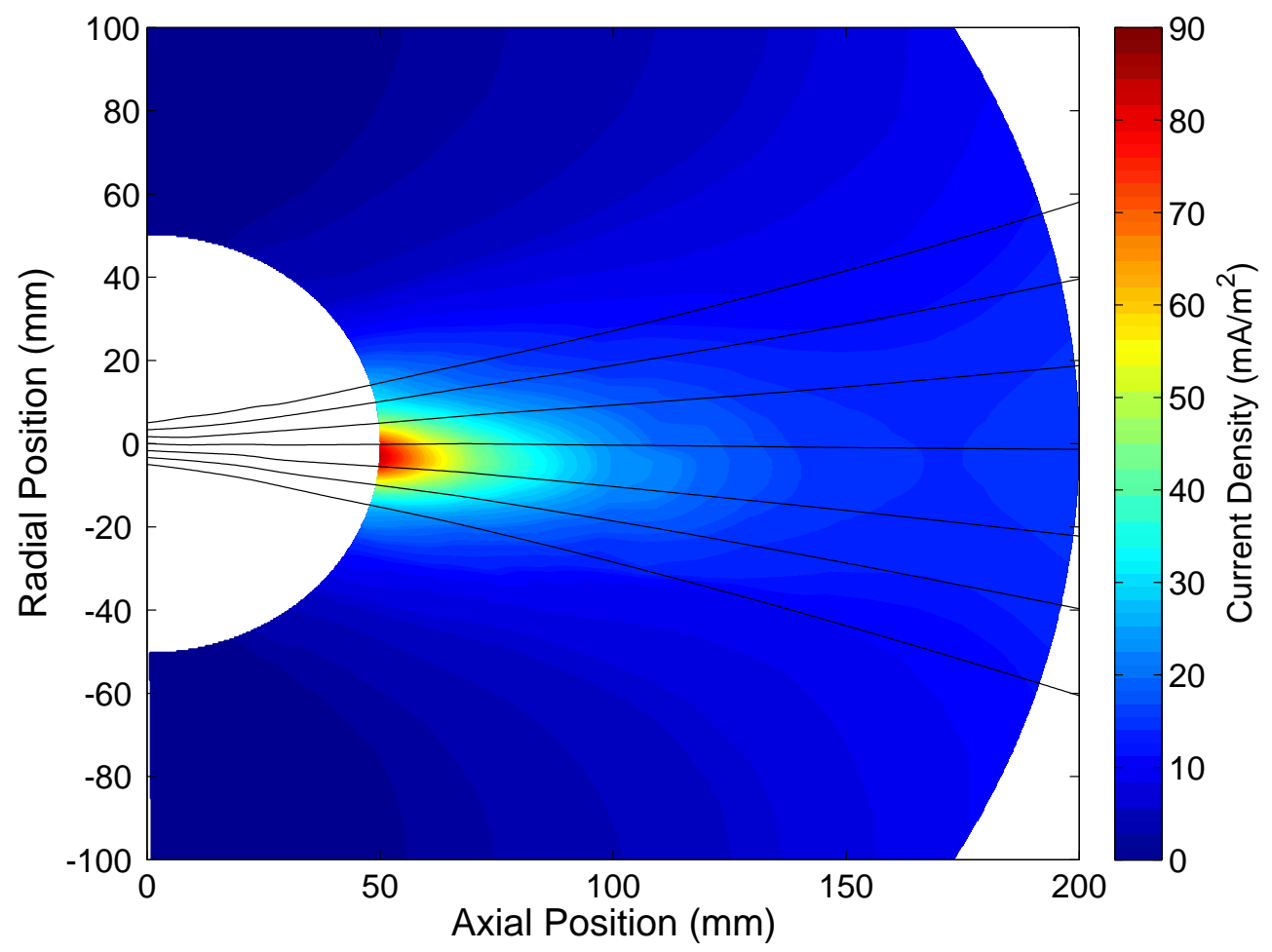

Figure 12: A contour map of the ion current density measured at a DC input power of $25.6 \mathrm{~W}$ with a magnetic field streamline overlay (black curves). The outermost streamlines define the edge of the magnetic nozzle at the thruster exit plane and are adjacent to the liner walls in the plasma generation region.

The relationship between the magnetic nozzle and the angular ion current density profiles was also investigated. As shown in Figure 12, qualitatively, the ion current closely follows the nozzle geometry, and the ion current density decreases as the magnetic nozzle cross sectional area increase. However, due to the high background pressure and the presence of frequent scattering collisions (discussed in more detail in the next section) effects such as plume detachment and the evolution of the beam could not be investigated. Future experiments will be conducted to investigate these phenomena in more depth. 


\section{Thrust Measurements}

Due to thermal runaway and the required cooling system to control the runaway CAT thrust was measured indirectly by a momentum target mounted to the $\mu N$ thrust stand. Prior to startup the thrust stand was calibrated and the cold gas thrust was measured by the thrust stand located at $15 \mathrm{~cm}$ downstream of the thruster exit plane, at each operating point. Each of the points shown in Figure 13 were measured while CAT was operating in the current-limited mode and the propellant flow rate was $3 \mathrm{mg} / \mathrm{s}$. CAT firing resulted in thrust values ranging from $150-200 \%$ of the corresponding cold gas thrust of $88 \mu \mathrm{N}$, with increasing thrust performance as input power was increased. Note that the reported power was the DC input power; the cooling system could not measure the electrical efficiency at all the thrust points reported.

However, these thrust performance measurements are misleading. With CAT operating at a propellant flow rate of $3 \mathrm{mg} / \mathrm{s}$ the Junior test facility could only sustain a back pressure of $4 \times 10^{-4}$ Torr, which results in significant scattering effects. An important scattering effect is charge exchange collisions (CEX) where an energetic ion collides with a cold background neutral, transferring its charge and resulting in an energetic neutral and a cold ion. For xenon the CEX collision cross section is approximately constant over the ion energy range of $1 \mathrm{~s}$ to $100 \mathrm{~s}$ of $\mathrm{eV},{ }^{28}$ or the ion energy range of many electric propulsion devices. As shown in Figure 14, as the background pressure, or the background neutral density, increases the CEX mean free path decreases significantly. For the CAT operational background pressure of $4 \times 10^{-4}$ Torr the CEX mean free path was $\sim 5 \mathrm{~cm}$, so an ion impacting the momentum target on the thrust stand, on average, underwent several CEX collisions. This implies that the measured thrust is due to ions that were not created and accelerated by CAT.

To obtain more meaningful thrust measurements the momentum target would need to be located within a CEX mean free path, or $5 \mathrm{~cm}$ for CAT operation at $3 \mathrm{mg} / \mathrm{s}$ in the Junior test facility. This is problematic because placing large objects within the plume can perturb the thruster operation. Another option would be to improve the pumping speed of the facility or repeat the experiment in a facility that can sustain back pressures below $5 \times 10^{-5}$ Torr, or CEX mean free paths $\leq 1 \mathrm{~m}$. Another consideration is the size of the momentum target, which must be larger than the magnetic nozzle cross sectional area at the location downstream. This is required to get accurate thrust measurements because, as shown in Figure 12 the majority of the ion current is contained within the magnetic nozzle field lines that are adjacent to the liner walls within the CAT. For the $10 \mathrm{~cm}$ diameter momentum paddle used in this experiment, this limits the position of the paddle to within $15 \mathrm{~cm}$ of the CAT exit plane. Overall, the thrust measurements reported demonstrate improvement over cold gas thruster performance, but do not yield actual performance metrics.

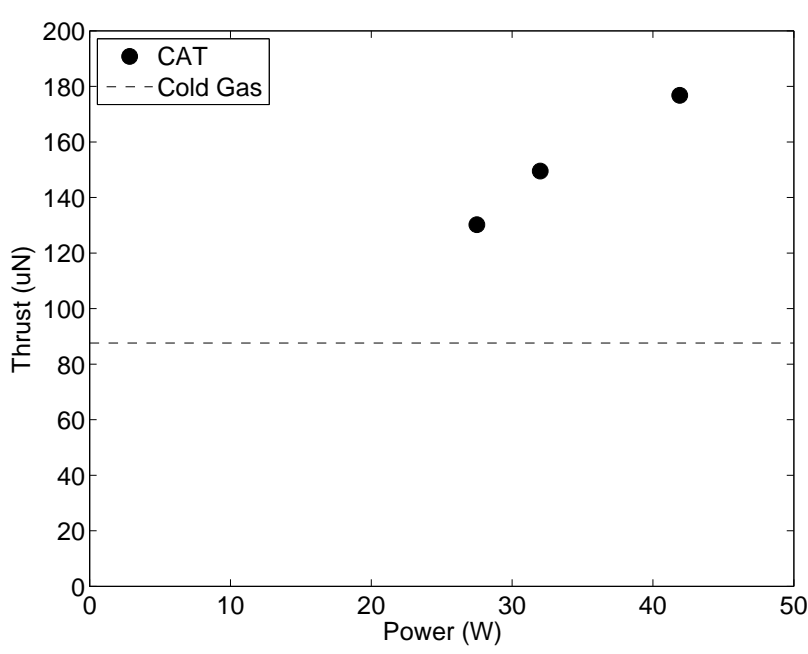

Figure 13: The measured thrust at various CAT power conditions at a propellant flow rate of $3 \mathrm{mg} / \mathrm{s}$ with the corresponding measured cold gas thrust.

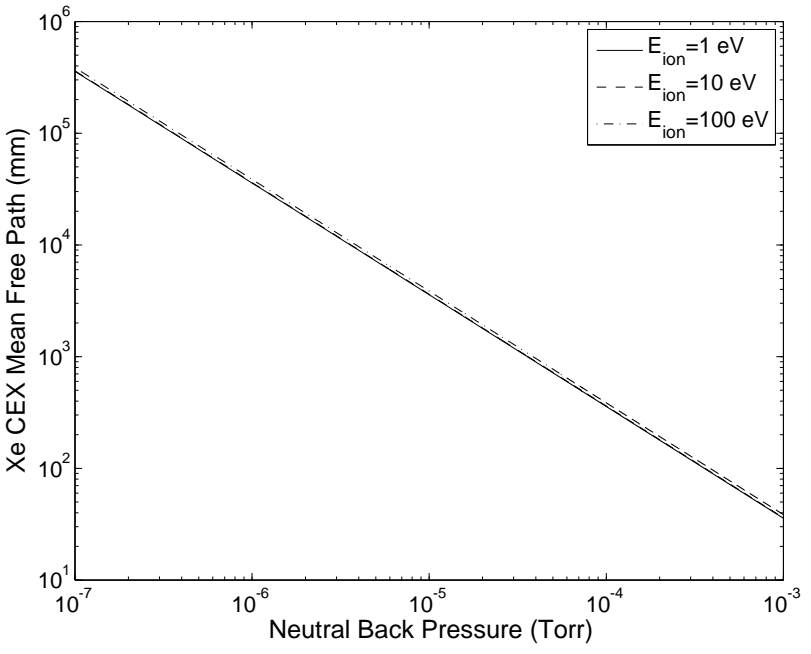

Figure 14: The charge exchange collision mean free path for xenon as a function of background pressure. 


\section{Future Work}

To date CAT operates at a fixed frequency of $9.96 \mathrm{MHz}$ and is not rematched after ignition, resulting in sub-optimal power deposition into the plasma. The next iteration of the PPU will include a variable frequency matching scheme to better couple power to the plasma, improving performance and reducing waste heat generation. In its current state CAT can be operated for extend periods of time ( $\geq 1$ minute) only if the closed loop cooling is active. Improved thermal management and heat rejection techniques will be incorporated, allowing for integration into a flight-like unit and direct thrust testing. To complement these thruster improvements the plume will be fully characterized, allowing for the study of the fundamental physics involved with mode transitions, plasma detachment, and possible instabilities. In parallel, a second CAT unit utilizing electromagnets will be created to optimize design and operating parameters and allow magnetic field effects to be investigated.

\section{Conclusion}

An integrated engineering unit of the CAT has been assembled and early testing results have been reported. Initial testing was limited to $\leq 1$ minute due to inadequate thermal management, resulting in thermal runaway. This was countered by a closed loop cooling system designed to yield an estimate of the waste heat rejected. Using the waste heat estimate the estimated electrical efficiency was consistently $\sim 50 \%$ across $25-30 \mathrm{~W}$ operating conditions. Measurements of plume oscillations were made to inform future probe design, yielding maximum peak to peak floating potential fluctuations of $150 \mathrm{mV}$, indicating $\mathrm{RF}$ compensation is not required. A parameter study of the angular ion current density profiles over propellant mass flow rates of $2.5-3.5 \mathrm{mg} / \mathrm{s}$ and power ranges of $25-36 \mathrm{~W}$ was conducted, indicating that decreasing propellant mass flow rate significantly increased the peak ion current density while increasing input power slightly increased peak current density. The plume divergence half angle was included in this parameter study, with decreasing propellant mass flow rate resulting in a significantly tighter beam while the beam tightness was insensitive to variations input power. A series of angular current density profiles were measured, indicating that the ion current closely follows the magnetic nozzle. Thrust was also indirectly measured using a momentum target, due to the required cooling connections. Improvement of $1.5-2 \times$ the cold gas thrust was observed, however these results were misleading because scattering collision were prevalent due to high operating background pressures. A combination of hardware improvements to CAT and facility changes will allow future experiments to overcome these obstacles and more fully investigate performance and plume phenomena.

\section{Acknowledgements}

This material is based upon work supported by the National Science Foundation Graduate Research Fellowship Program under Grant No. DGE 1256260, the National Aeronautics and Space Administration (NASA) under Award No. NNX14AD71G, and the Defense Advanced Research Projects Agency (DARPA) under NASA Contract Number NNA15BA42C. The authors would like to thank Phase Four, Inc. for their assistance and insights. Thank you to the members of the Plasmadynamics and Electric Propulsion Laboratory for their insightful discussion concerning this research.

\section{References}

${ }^{1}$ Folta, D., Dichmann, D., Clark, P., Haapala, A., and Howell, K., "Lunar Cube Transfer Trajectory Options," 25th AAS/AIAA Space Flight Mechanics Meeting, 2015.

${ }^{2}$ Rhee, M., Zakrzwski, C., and Thomas, M., "Highlights of Nanosatellite Propulsion Development Program at NASAGoddard Space Flight Center," Small Satellite Conference, 2000.

${ }^{3}$ Schmuland, D., Masse, R., and Sota, C., "Hydrazine propulsion module for cubesats," Small Satellite Conference, 2011.

${ }^{4}$ Schmuland, D. T., Carpenter, C., and Masse, R. K., "Mission Applications of the MRS-142 CubeSat High-Impulse Adaptable Monopropellant Propulsion System (CHAMPS)," No. 2012-4269, 2012.

${ }^{5}$ Zondervan, K., Fuller, J., Rowen, D., Hardy, B., Kobel, C., Chen, S.-H., Morrison, P., Smith, T., and Kremer, A., "CubeSat Solid Rocket Motor Propulsion Systems providing Delta-Vs greater than $500 \mathrm{~m} / \mathrm{s}$," Small Satellite Conference, 2014.

${ }^{6}$ Bertino-Reibstein, A. and Wuerl, A., "Development of a Warm-Gas Butane System for Microsatellite Propulsion," Small Satellite Conference, 2013.

${ }^{7}$ Dinardi, A. and Persson, M., "High performance green propulsion (HPGP): a flight-proven capability and cost game- 
changer for small and secondary satellites," Small Satellite Conference, 2012.

${ }^{8}$ Dyer, J., Dinardi, A., and Anflo, K., "First Implementation of High Performance Green Propulsion in a Constellation of Small Satellites," Small Satellite Conference, 2013.

${ }^{9}$ Morris, D. and Noble, R., "CubeSat advanced technology propulsion system concept," Small Satellite Conference, 2014.

${ }^{10}$ Orr, N., Eyer, J., Larouche, B., and Zee, R., "Precision formation flight: the CanX-4 and CanX-5 dual nanosatellite mission," Small Satellite Conference, 2007.

${ }^{11}$ Platt, D., "A monopropellant milli-Newton thruster system for attitude control of nanosatellites," Small Satellite Conference, 2002.

${ }^{12}$ Zhuang, T., Shashurin, A., Beilis, I., and Keidar, M., "Ion velocities in a micro-cathode arc thruster," Vol. 19, 2012, p. 063501 .

${ }^{13}$ Trezzolani, F., Fabris, A. L., Pavarin, D., Selmo, A., and Manente, M., "Low Power Radio-Frequency Plasma Thruster Development and Testing," IEPC-2013-153, 33rd International Electric Propulsion Conference, 2013.

${ }^{14}$ Spence, D., Ehrbar, E., Rosenblad, N., Demmons, N., Roy, T., Hoffman, S., Williams, D., Hruby, V., and Tocci, C., "Electrospray propulsion systems for small satellites," Small Satellite Conference, 2013.

${ }^{15}$ Shashurin, A., Keidar, M., and Zhuang, T., "Comparative analysis of micro-cathode arc thruster performance," IEPC2013-389, 33rd International Electric Propulsion Conference, 2013.

${ }^{16}$ Martel, F., Perna, L., and Lozano, P., "Miniature Ion Electrospray Thrusters and Performance Test on CubeSats," Small Satellite Conference, 2012.

${ }^{17}$ Kronhaus, I., Schilling, K., Jayakumar, S., Kramer, A., Pietzka, M., and Schein, J., "Design of the UWE-4 Picosatellite Orbit Control System using Vacuum-Arc-Thrusters," IEPC-2013-195, Proceedings of the 33rd International Electric Propulsion Conference, 2013.

${ }^{18}$ Ketsdever, A. D., Lee, R. H., and Lilly, T. C., "Performance testing of a microfabricated propulsion system for nanosatellite applications," Vol. 15, 2005, p. 2254.

${ }^{19}$ Keidar, M., Haque, S., Zhuang, T., Shashurin, A., Chiu, D., Teel, G., Agasid, E., Tintore, O., and Uribe, E., "Microcathode arc thruster for PhoneSat propulsion," Small Satellite Conference, 2013.

${ }^{20}$ Fuchikami, S., Nakamoto, M., Toyoda, K., and Cho, M., "Development of Vacuum Arc Thruster for Nano-Satellite," IEPC-2013-264, 33rd International Electric Propulsion Conference, Washington DC, USA, 2013.

${ }^{21}$ Coletti, M., Guarducci, F., and Gabriel, S., "A micro PPT for Cubesat application: Design and preliminary experimental results," Vol. 69, 2011, pp. 200-208.

${ }^{22}$ Coletti, M., Ciaralli, S., and Gabriel, S. B., "PPT Development for Nanosatellite Applications: Experimental Results," Vol. 43, 2015, pp. 218-225.

${ }^{23}$ Ciaralli, S., Coletti, M., and Gabriel, S., "Performance and lifetime testing of a pulsed plasma thruster for Cubesat applications," Vol. 47, December 2015, pp. 291-298.

${ }^{24}$ Collard, T. A., Sheehan, J., and Gallimore, A. D., "Pressurized Xenon Propellant Management System for the CubeSat Ambipolar Thruster," IEPC-2015-364, 34th International Electric Propulsion Conference, Hyogo-Kobe, Japan, 2015.

${ }^{25}$ Tsay, M., Frongillo, J., Hohman, K., and Malphrus, B. K., "LunarCube: A Deep Space $6 \mathrm{U}$ CubeSat with Mission Enabling Ion Propulsion Technology," 2015.

${ }^{26}$ Brown, D. L. and Gallimore, A. D., "Evaluation of ion collection area in Faraday probes," Vol. 81, 2010, p. 063504.

${ }^{27}$ Brown, D. L., Larson, v. W., Beal, B. E., and Gallimore, A. D., "Methodology and historical perspective of a Hall thruster efficiency analysis," Vol. 25, 2009, pp. 1163-1177.

${ }^{28}$ Piscitelli, D., Phelps, A. V., de Urquijo, J., Basurto, E., and Pitchford, L. C., "Ion mobilities in Xe/Ne and other rare-gas mixtures," Phys. Rev. E, Vol. 68, Oct 2003, pp. 046408. 
This article has been cited by:

1. Timothy A. Collard, JP Sheehan, Benjamin A. Jorns. A Numerical Examination of the Performance of Small Magnetic Nozzle Thrusters . [Citation] [PDF] [PDF Plus]

2. Kristina Lemmer. 2017. Propulsion for CubeSats. Acta Astronautica 134, 231-243. [Crossref] 